\title{
EFECTOS DE LA MEDICIÓN DE ONDAS SUPERFICIALES CONSIDERANDO DIFERENTES GEOMETRÍAS Y CONDICIONES DE EMPLAZAMIENTO CON BASE EN LA METODOLOGÍA FRECUENCIA-NÚMERO DE ONDA (F-K)
}

\author{
EFFECTS OF SURFACE WAVE RECORDINGS CONSIDERING DIFFERENT \\ GEOMETRIES AND SITE CONDITIONS USING FRECUENCY-WAVE \\ NUMBER TECHNIQUE (F-K)
}

\author{
José P. Echeverría ${ }^{1 *} \&$ Víctor Schmidt-Díaz ${ }^{2}$ \\ ${ }^{1}$ Laboratorio de Ingeniería Sísmica. Nivel 3-A Facultad de Ingeniería, \\ Universidad de Costa Rica, 2060, San Pedro de Montes de Oca. \\ ${ }^{2}$ Laboratorio de Ingeniería Sísmica. Nivel 3-A Facultad de Ingeniería, \\ Universidad de Costa Rica, 2060, San Pedro de Montes de Oca. \\ *Autor para contacto:jpecheve@gmail.com
}

(Recibido: 07/10/2015; aceptado: 11/03/2016)

\begin{abstract}
Surface wave recordings are a novel technique for the seismic classification of sites regarding dynamic properties. Therefore, an evaluation of the results of ambient vibrations with different geometrical configurations and site conditions is needed. This work is a study of the effect of the configurations, number of sensors, dimension of the radius and time of recording in the methodology of arrays. In this paper, three sites were selected for the application of surface wave recordings: Parque Metropolitano La Sabana, Instalaciones Deportivas de la Universidad de Costa Rica and the Estadio Municipal de Palmares. In each of these, geometrical variations in the array disposition were studied using the Frequency-Wavenumber (F-K) technique. The array with the best results using the F-K technique was the circular configuration, combining large and a small opening. It is recommended a minimum time of recording of 30 minutes to obtain the needed information from the ambient vibrations, as well.

Keywords: Surface waves, dispersion curve, arrays, frequency-wave number (F-K), ambient vibrations.

RESUMEN: Se entiende que la medición de ondas superficiales es una forma novedosa para la clasificación de sitios de cimentación, con base en parámetros dinámicos como el periodo fundamental del sitio y Vs30. Por lo tanto, se requiere evaluar los resultados de la medición de vibraciones ambientales usando diferentes configuraciones geométricas y condiciones de emplazamiento. En la presente publicación, se seleccionaron tres sitios para la aplicación de la prueba: El Parque Metropolitano La Sabana, Las Instalaciones Deportivas de la Universidad de Costa Rica y el Estadio
\end{abstract}

ECHEVERRÍA, J. P. \& SCHMIDT-DÍAZ, V., 2016: Efectos de la medición de ondas superficiales considerando diferentes geometrías y condiciones de emplazamiento con base en la metodología frecuencia-número de onda (F-K).- Rev. Geol. Amér. Central, 55: 101-114, DOI: 10.15517/rgac.v55i0.27065 
Municipal de Palmares. En cada uno de ellos, se estudió el efecto de la configuración, número de sensores, apertura y tiempo de medición según la metodología de arreglos de sensores con el uso de la técnica Frecuencia-Número de Onda (F-K). Se logró concluir que las configuraciones de arreglos de sensores circulares, que combinan radios grandes y pequeños, generan los mejores resultados según la metodología F-K. Se recomendó un tiempo mínimo de registro de 30 minutos para lograr obtener la suficiente cantidad de información de las vibraciones ambientes por medio de arreglos de sensores en el sitio.

Palabras clave: Ondas superficiales, curva de dispersión, arreglos de sensores, frecuencia-número de onda (F-K), vibraciones ambientales.

\section{INTRODUCCIÓN}

Es sabido y documentado que los terremotos más fuertes han sido la causa de miles de personas muertas y afectadas alrededor del mundo. Varios de los centros de población de nuestro país y el mundo se ubican en depósitos de suelos blandos, los cuales tienen una relación directa con el aumento de la intensidad sísmica de un terremoto, como lo sucedido en México de 1985 (Sauter, 1989). Cuando una onda sísmica atraviesa un depósito de suelos blandos, se genera un aumento de su respuesta respecto a su comportamiento en roca. Esto es llamado amplificación sísmica, que depende de geología superficial y de las propiedades mecánicas del suelo.

Para determinar la amplificación sísmica de un sitio es fundamental conocer el tipo de suelo que subyace. Según normas nacionales e internacionales, la clasificación de un emplazamiento se correlaciona con parámetros dinámicos como el periodo fundamental (T0) y la velocidad de onda cortante de los 30 metros superficiales (Vs30), entre otros. Con base en la medición de ondas superficiales, se puede calcular dichos parámetros mediante el uso de técnicas empíricas como los arreglos de sensores. Por lo tanto, estudios en la línea de la calibración de métodos de medición de ondas superficiales son de gran relevancia para la zonificación sísmica del país, ya que permiten definir metodologías de trabajo más precisas y eficientes.

Debido a que hasta hace muy poco tiempo se emplean este tipo de técnicas en el país (Schmidt, 2014), no se ha realizado un análisis de la forma en que diferentes geometrías y condiciones de emplazamiento pueden variar los datos obtenidos, que pueden ocasionar diferencias en los resultados de la caracterización de sitio para una misma ubicación. Esta publicación se plantea como un insumo necesario para poder optimizar las pruebas en cuanto a geometrías, cantidad de sensores, tiempos de medición y apertura del arreglo.

\section{MARCO TEÓRICO}

\section{El método de arreglo de sensores}

El uso de sensores colocados en serie, llamados arreglos de sensores, ha resultado ser un atractivo método empírico para la determinación de las velocidades de onda cortante y los espesores de estratos a partir de mediciones de ruido hechas "in situ" (SESAME, 2005). La principal ventaja que tiene con respecto a metodologías similares es que no necesita de fuentes activas que generen los frentes de ondas, las cuales generalmente son destructivas o invasivas. Por el contrario la metodología de arreglos de sensores utiliza generación pasiva de ruido ambiental, como lo son los microsismos y microtemblores, por lo que resulta ser una técnica sencilla en su aplicación

Al colocar el arreglo de sensores, se asume que las vibraciones ambientales están compuestas en su mayoría por ondas superficiales (SESAME, 2004) y que el subsuelo se compone de estratos homogéneos y horizontalmente dispuestos, con la suficiente impedancia entre un estrato y su adyacente. En medios unidimensionales y heterogéneos, las ondas superficiales son dispersivas, por lo que muestran variaciones de velocidad aparente según la frecuencia" (Schmidt, 2010). El contenido frecuencial del registro de los microtremores se distribuye sobre un rango muy amplio, por lo 
que la medición de ondas superficiales por el método de arreglos de sensores es una opción muy interesante como complemento a las que requieren el uso de fuentes activas.

En la obtención de curvas de dispersión y perfiles de suelo, se plantean dos formas de orientación del problema: a) proceso directo: si se parte de un perfil de suelo conocido y un de frente de ondas que lo atraviesa, es posible conocer la curva de dispersión asociada, en este caso el procesado suele ser analítico o numérico y por lo tanto tiene solución única (Schmidt, 2010); b) proceso inverso: partiendo de un registro de sensores en serie (arreglos de sensores) se puede construir una curva de dispersión para el medio y mediante un proceso de inversión de la curva, con base en un algoritmo de aproximaciones sucesivas, se llega a encontrar un perfil de suelo que genere el menor error asociado. En el problema inverso, se tiene la particularidad de que existen muchos métodos para llegar a la solución y normalmente se generan familias de éstas, cada una con su incertidumbre y su error asociado. En el siguiente diagrama (Fig. 1) se ilustran los procesos directo e inverso mencionados anteriormente:

\section{Metodología de la Frecuencia-Número de Onda (F-K)}

La metodología de la Frecuencia-Número de Onda (F-K) es una de las técnicas que existe para el manejo de los registros de ondas superficiales obtenidos de los arreglos de sensores. La metodología se basa en la construcción de una curva de dispersión con base en la máxima respuesta del arreglo para cada frecuencia en particular, dentro del plano kx-ky, que corresponde a los números de onda en componentes xy del plano horizontal del arreglo de sensores.

El método calcula la semblanza, como la razón entre la salida del arreglo (beam power) y la energía de entrada, ya que esta provee una medida de la energía que llega a un punto. En la práctica, la localización de la máxima semblanza se hace en el plano kx-ky que provee una estimación de la velocidad y el azimut de las ondas que viajan a través del arreglo, que se determina donde se observa la máxima energía (Wathelet, 2007).
Mediante los tiempos de arribo de las ondas a los diferentes sensores del arreglo, se logra construir la máxima semblanza del mismo.

El proceso que es realizado para todo el rango de frecuencias, da como resultado la curva de dispersión del medio. En esta curva, los puntos obtenidos para las altas frecuencias brindan información de las capas más superficiales, mientras que las frecuencias bajas se necesitan para llegar a profundidades mayores del medio. De esta forma, los arreglos de sensores con menor apertura de radio brindan información de frecuencias más altas, con lo que se logra caracterizar las capas más someras, y los arreglos con aperturas mayores generan mejor información de frecuencias bajas y a su vez capas más profundas.

\section{DESCRIPCIÓN DE LAS PRUEBAS REALIZADAS}

En esta sección se presenta la metodología general seguida en las pruebas, explicando la apertura de los arreglos geométricos, la configuración y cantidad de sensores utilizados y los tiempos de registro de cada una de las mediciones. Se realizaron registros de ondas superficiales en tres sitios distintos para tener criterios más concluyentes acerca de los resultados obtenidos. Estos sitios son: Parque Metropolitano La Sabana, Instalaciones Deportivas de la Universidad de Costa Rica y el Estadio Municipal de Palmares.

Para la medición de ondas superficiales, se utilizó el equipo proporcionado por el Laboratorio de Ingeniería Sísmica (LIS). El LIS facilitó el equipo de medición de vibraciones ambientales conformado por: ocho sismógrafos LE-3D/20s de la marca Lennartz, sensores que registran las ondas en términos de velocidad $(\mathrm{cm} / \mathrm{s})$, y cuatro digitalizadores 130S-1 de la marca REFTEK mediante los cuales se graba la señal registrada por los sismógrafos. El tiempo es controlado por medio de un sistema de posicionamiento global (GPS), conectado a los digitalizadores. Es importante destacar que el equipo tiene una limitante en cuanto a la apertura de los arreglos, ya que la distancia máxima que puede ser cubierta por los cables es de $50 \mathrm{~m}$, lo que implica un diámetro máximo del arreglo de $100 \mathrm{~m}$. 


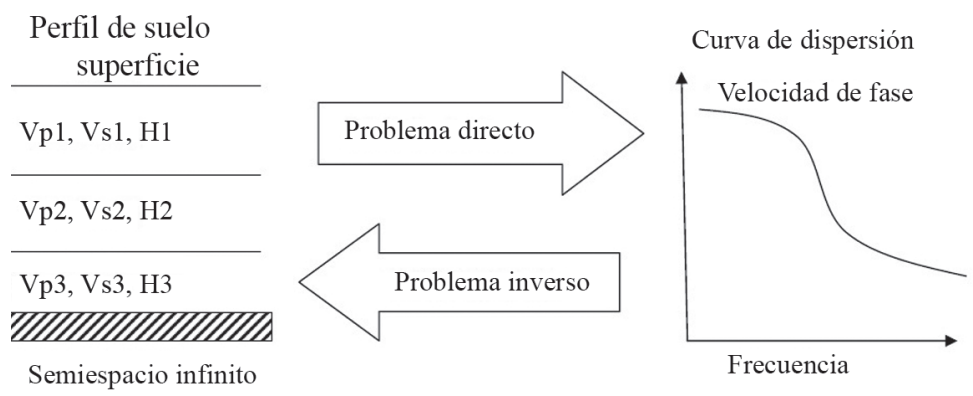

Fig. 1: Esquema del proceso directo e inverso de dispersión y perfiles de suelo (Schmidt, 2010).

\section{Parque Metropolitano La Sabana}

Estas mediciones se ubicaron en una de las canchas de béisbol al sur del Estadio Nacional, dentro del Parque Metropolitano La Sabana. Fueron realizadas el 25 de setiembre del 2013 a partir de las 9:00 a.m. Se presentan los rangos de coordenadas y elevaciones para la cancha de béisbol donde se realizaron las mediciones de ondas superficiales en La Sabana:

Latitud: $9^{\circ} 56^{\prime} 03^{\prime \prime} \mathrm{N}-9^{\circ} 56^{\prime} 06^{\prime \prime} \mathrm{N}$

Longitud: $84^{\circ} 06^{\prime} 24^{\prime}$ ' O - $84^{\circ} 06^{\prime} 27^{\prime \prime} \mathrm{O}$

Elevación: 1128-1132 msnm

En el cuadro 1 se detallan las condiciones de medición de cada prueba realizada en el Parque Metropolitano de La Sabana.

\section{Instalaciones Deportivas de la Universidad de Costa Rica (UCR)}

Estos registros se llevaron a cabo en una canchas de béisbol del sector norte de las Instalaciones Deportivas de la Sede Central de la UCR, en Finca 3, en el distrito de Mercedes de Montes de Oca. Las mismas se llevaron a cabo durante los días el 11 de setiembre y 30 de octubre del 2013 y el 17 de enero del 2014. Se presentan los rangos de coordenadas y elevaciones para la cancha de béisbol donde se realizaron las mediciones de vibraciones ambientales en las Instalaciones Deportivas de la UCR:

Latitud: $9^{\circ} 56^{\prime} 41,5^{\prime \prime} \mathrm{N}-9^{\circ} 56^{\prime} 45,5^{\prime \prime} \mathrm{N}$

Longitud: $84^{\circ} 02^{\prime} 46,5^{\prime}$ ' O - 84 $02^{\circ} 49,5^{\prime \prime} \mathrm{O}$

Elevación: 1221-1225 msnm
En el cuadro 2 se detallan las condiciones de medición de cada prueba realizada en las Instalaciones Deportivas de la UCR en Sabanilla.

\section{Estadio Municipal Jorge Palmareño Solís de Palamares, Alajuela}

Estas mediciones se situaron en una de la cancha de fútbol del Estadio Municipal Jorge Palmareño Solís de Palmares, Alajuela. Las pruebas se llevaron a cabo durante el 29 de enero del 2014. Se presentan los rangos de coordenadas y elevaciones para la cancha de futbol donde se realizaron las mediciones en el Estadio Municipal de Palmares:

Latitud: $10^{\circ} 03^{\prime} 26^{\prime \prime} \mathrm{N}-10^{\circ} 03^{\prime} 30^{\prime \prime} \mathrm{N}$

Longitud: $84^{\circ} 25^{\prime} 46^{\prime \prime} \mathrm{O}-84^{\circ} 25^{\prime} 49^{\prime \prime} \mathrm{O}$

Elevación: 1018-1020 msnm

En el cuadro 3 se detallan las condiciones de medición de cada prueba realizada en el Estadio Municipal de Palmares, Alajuela.

\section{DESCRIPCIÓN LITOLÓGICA SIMPLIFICADA DE LOS SITIOS}

El cuadro 4 muestra una descripción geotécnica simplificada de los perfiles de suelo según la información disponible en SENARA (Servicio Nacional de Aguas Subterráneas, Riego y Avenamiento), para los 3 sitios considerados. 
Cuadro 1

Descripción general de las mediciones de ondas superficiales realizadas en el Parque Metropolitano La Sabana, San José.

\begin{tabular}{|c|c|c|}
\hline Prueba & Radio de apertura (m) & Tiempo de medición (min) \\
\hline \multirow{3}{*}{ Configuración Circular: 8 Sensores } & 46 & 60 \\
\hline & 23 & 60 \\
\hline & 10 & 30 \\
\hline Configuración Triangular: 7 Sensores & $\begin{array}{l}46 \text { externo } \\
15 \text { interno }\end{array}$ & 30 \\
\hline \multirow{2}{*}{ Configuración Circular: 6 Sensores } & 45 & 30 \\
\hline & 22,5 & 30 \\
\hline
\end{tabular}

\section{Cuadro 2}

Descripción general de las mediciones de ondas superficiales realizadas en las Instalaciones Deportivas de la UCR.

\begin{tabular}{ccc}
\hline Prueba & Radio de apertura $(\mathrm{m})$ & Tiempo de medición $(\mathrm{min})$ \\
\hline & 45 & 60 \\
Configuración Circular: 8 Sensores & 22,5 & 60 \\
& 10 & 30 \\
Configuración Triangular: 7 Sensores & 46 externo & 30 \\
Configuración Circular: 6 Sensores & 15 interno & 30 \\
\hline
\end{tabular}

\section{Cuadro 3}

Descripción general de las mediciones de ondas superficiales realizadas en el Estadio Municipal Jorge Palmareño Solís de Palmares, Alajuela.

\begin{tabular}{|c|c|c|}
\hline Prueba & Radio de apertura (m) & Tiempo de medición (min) \\
\hline \multirow{3}{*}{ Configuración Circular: 8 Sensores } & 40 & 60 \\
\hline & 22,5 & 60 \\
\hline & 10 & 30 \\
\hline Configuración Triangular: 7 Sensores & 46 externo & 30 \\
\hline \multirow{2}{*}{ Configuración Circular: 6 Sensores } & 45 & 30 \\
\hline & 22,5 & 30 \\
\hline
\end{tabular}

Se observa que en los 3 casos, en los primeros 10 metros existe la presencia de arcilla y seguidamente un contraste significativo de materiales, lo que deberá implicar un aumento significativo de la velocidad de onda cortante (Vs). Solo el caso de Palmares muestra en profundidad la presencia de arcillas, antecedidas por materiales más competentes, lo que seguramente implica una inversión de Vs.

\section{RESULTADOS}

\section{Tiempo de medición en los registros}

En esta sección se estudiará el efecto que tiene el tiempo total de medición de la prueba en la curva de dispersión obtenida por medio de la metodología de F-K. Con esto se desea definir 
Cuadro 4

Descripciones litológicas simplificadas de cada emplazamiento según el SENARA.

\begin{tabular}{|c|c|c|}
\hline Emplazamiento & Profundidad (m) & Descripción litológica según pozos disponibles \\
\hline \multirow{5}{*}{ Palmares } & $0-6$ & Arcilla color café claro, muy plástica \\
\hline & $6-17$ & Toba fracturada color gris \\
\hline & $17-30$ & $\begin{array}{l}\text { Material arenoso (contiene fragmentos de lava, cuarzo, etc,) con arcilla, } \\
\text { aparentemente se trata de un depósito lacustre }\end{array}$ \\
\hline & $30-36$ & Arcilla con arena fina, color café \\
\hline & $36-40$ & Arcilla color gris \\
\hline \multirow{2}{*}{ Universidad de Costa Rica } & $0-10$ & Arcilla café \\
\hline & $10-30$ & Lahar, Contiene arenas, arcilla, fragmentos lávicos y caolinizados \\
\hline \multirow{3}{*}{ La Sabana } & $0-13$ & Arcilla, color café claro, Cenizas compactadas, de grano muy fino \\
\hline & $13-31$ & $\begin{array}{l}\text { Lavina, color café claro, con algún contenido arcilloso y una serie de } \\
\text { fragmentos de otras rocas, Entre estos fragmentos hay lavas, piroclastos, } \\
\text { fenocristales, etc. }\end{array}$ \\
\hline & $31-40$ & $\begin{array}{l}\text { Toba color gris ceniciento, Con cristales de anfíboles y feldespatos en una } \\
\text { matriz de ceniza }\end{array}$ \\
\hline
\end{tabular}

tiempos mínimos de registro que se deben cumplir para obtener información confiable y precisa del suelo subyacente. Es importante destacar que el tiempo de medición total debe cumplir con el tamaño de ventana mínimo y la cantidad de ventanas que se requieren, según las recomendaciones de (Bard, 2004). Con una duración de ventana de $30 \mathrm{~s}$ se cumple con un mínimo de diez periodos fundamentales (T0), recomendado de acuerdo con la referencia citada y para un número de 20 ventanas, se debe cumplir como mínimo con 10 minutos de medición $(20 * 30 \mathrm{~s}=600 \mathrm{~s}=10 \mathrm{~min})$. En este caso, se desea comparar y contrastar los resultados experimentales con los que se recomiendan en la bibliografía.

En la figura 2 se presentan los resultados de las curva de dispersión según la metodología F-K en las Instalaciones Deportivas de la Universidad de Costa Rica para la configuración circular de ocho sensores (siete perimetrales y uno central) para mediciones de una hora, $30 \mathrm{~min}, 15 \mathrm{~min}$ y 5 min. En los otros dos sitios considerados, los resultados fueron muy semejantes.

Debido a que el registro de las ondas, que son continuas en el tiempo y en espacio, se hace a partir de un número finito de sensores, existen límites en cuanto al rango de frecuencias en las cuales es posible tener certeza de la lectura de las velocidades o lentitudes correspondientes a la dispersión. Las curvas que definen los límites teóricos que se observan en las siguientes figuras (Fig.2), representan el rango de valores dentro de los cuales la curva de dispersión es confiable, de acuerdo con Wathelet (2007). Los denominados máximos corresponden a zonas de la curva en las que la relación lentitud-frecuencia aproximadamente confiable, mientras que los rangos seguros representan regiones en las cuales la curva de dispersión lo es totalmente. Los límites superiores (altas frecuencias) evitan el efecto de aliasing (fenómeno que se da al registrar un proceso continuo en el espacio con un número de instrumentos finito) y que condiciona la mínima longitud de onda que se puede considerar de forma confiable. Por otro lado, los límites inferiores corresponden a la resolución: máxima longitud de onda que es posible observar a partir de la configuración de sensores escogida.

En cuanto al efecto del tiempo total de medición, en general en los tres sitios y bajo las diferentes configuraciones utilizadas se nota que al disminuir el tiempo de medición, la curva pierde resolución, o sea que se vuelve más difícil observar su forma. Al comparar las mediciones de una hora, la de treinta minutos y la de quince 

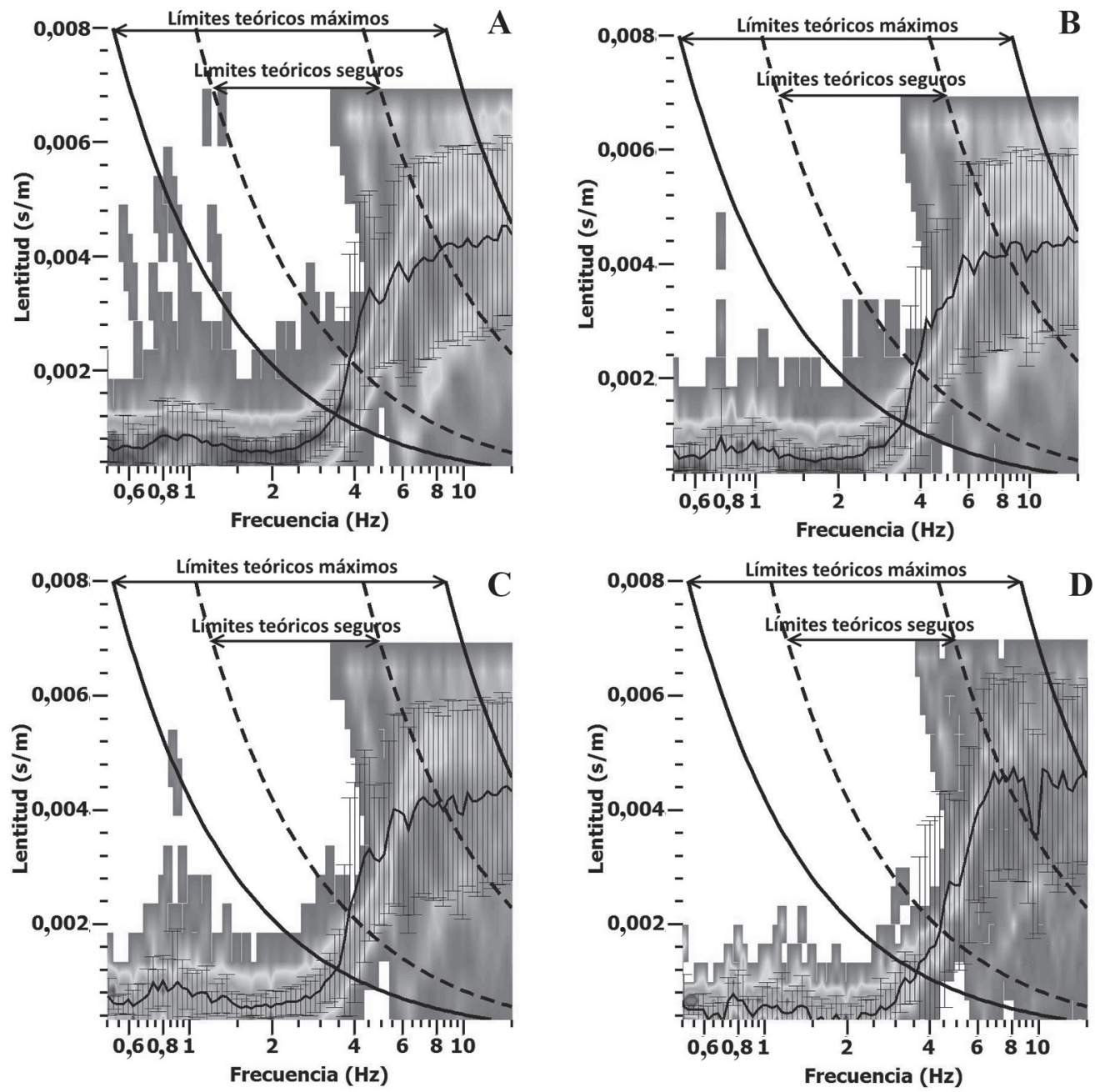

Fig. 2: Curva de dispersión del arreglo circular de 8 sensores con radio de $45 \mathrm{~m}$ en las Instalaciones Deportivas de la UCR, medición con duración de (a) $60 \mathrm{~min}$, (b) $30 \mathrm{~min}$, (c) $15 \mathrm{~min}$ y (d) 5 min.

minutos no se encuentran diferencias significativas sobre la curva de dispersión en la mayor parte del rango de frecuencias permitido por la respuesta teórica del arreglo (líneas curvas en la Fig. 2). Sin embargo, se observa que el tiempo de medición tiene un efecto más visible en la desviación estándar (definida por la claridad de curva en forma de "S" de dispersión y las barras verticales por encima y por debajo de la traza negra promedio). En general, aunque las curvas de una hora, treinta y quince minutos sigan aproximadamente la misma forma, es claro que la desvia- ción estándar de la medición disminuye al aumentar el tiempo de registro. Es importante resaltar que la estimación de esta desviación es esencial para la obtención de los modelos de perfiles de Vs, ya que en gran medida define los límites que tiene el algoritmo de inversión para generar soluciones que se apeguen más o menos al promedio obtenido según la forma de la curva. Por ejemplo, desviaciones estándar grandes permiten soluciones más alejadas del promedio pero genera modelos menos realistas.

Con respecto a las curvas de dispersión generadas para la medición de cinco minutos, 
se observa la poca cantidad puntos que se despliegan en la gran mayoría de frecuencias. En la figura 2 (d) se aprecia que en las altas frecuencias, dentro de los límites teóricos, se pierde resolución en la curva y se tiende a confundir con el efecto de "aliasing", por lo que es justificable que tenga una desviación estándar muy alta debido a la gran dispersión de los puntos.

\section{Configuración geométrica del arreglo de sensores}

En materia de configuraciones geométricas de los arreglos de sensores, existen múltiples variaciones que se pueden estudiar: arreglo cartesiano, arreglo circular con y sin sensor central, espiral, en estrella, entre otros; la única restricción impuesta por la metodología es conocer la ubicación relativa de los sensores respecto a punto (o eje) de referencia. Sin embargo, según estudios realizados por diversos autores (SESAME, 2005), las configuraciones que han dado mejores resultados en términos de la respuesta teórica del arreglo (definición del límite superior e inferior de frecuencias donde la información obtenida de la curva de dispersión es confiable) son los arreglos: circulares, en estrella y en espiral.

En esta etapa, se decidió realizar el estudio con configuraciones circulares y triangulares, debido a que son las más comunes y fáciles de aplicar en el campo. La configuración triangular está compuesta por dos arreglos en forma de triángulos concéntricos registrando al mismo tiempo, con lados de distinta longitud y con un desfase angular para que logren una adecuada distribución azimutal entre ellos. En el caso de los arreglos circulares, se utilizaron arreglos de ocho y seis sensores, en ambos casos colocando uno central y el resto perimetrales en forma de círculo. En la figura 3 se muestra la distribución en el plano horizontal de los sensores para los arreglos circulares de ocho (Fig. 3a) y siete sensores (Fig. 3b), así como el arreglo triangular de siete sensores (dos triángulos concéntricos, Fig. 3c). La información de los arreglos utilizados en cada uno de los tres sitios (apertura, tiempo de medición, etc.) se encuentra en los cuadros 1,2 y 3 .
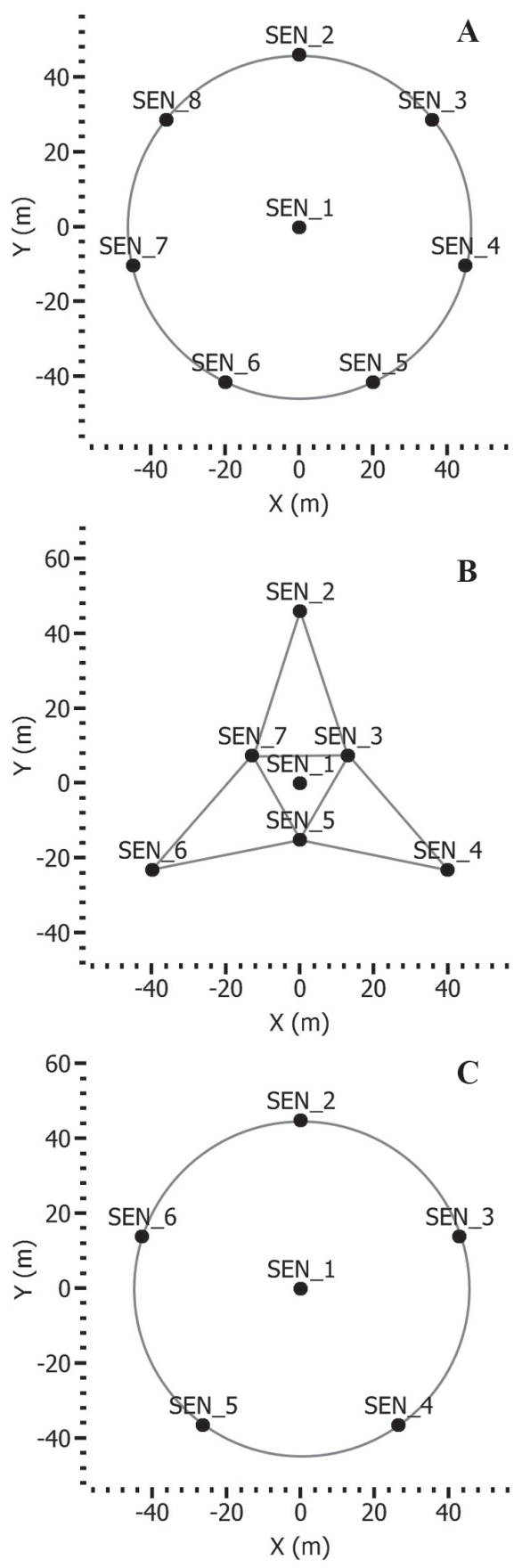

Fig. 3: Ubicación en planta de arreglos geométricos (a) circular de ocho sensores, (b) triangular, que corresponde a dos triángulos de tres sensores cada uno más uno central y (c) circular de siete sensores. 
Se procedió a realizar un estudio de la respuesta teórica de cada una de las configuraciones de los arreglos, así como de las curvas de dispersión. Se quiere comprobar si un arreglo en forma de triángulo (con dos triángulos concéntricos de radio grande y pequeño que registran simultáneamente) brinda información similar a la que da la combinación de dos arreglos circulares de radio distinto registrando en tiempos distintos. Por lo tanto, para las configuraciones circulares de ocho y seis sensores, se realizó un promedio de las curvas de dispersión obtenidas para la apertura mayor y la menor. Finalmente, en cada lugar de medición se obtuvieron tres límites teóricos y tres curvas de dispersión: promedio del arreglo circular de ocho sensores, promedio arreglo circular de seis sensores y arreglo triangular de siete sensores y se compararon en la misma figura.

En figura 4 se presentan los resultados de las curvas de dispersión generadas en el Parque Metropolitano La Sabana para la combinación de arreglos de ocho y seis sensores con radio grande y pequeño (de $46 \mathrm{~m}$ y $23 \mathrm{~m}$, en ambos casos respectivamente); y para el arreglo triangular de siete sensores registrando simultáneamente (dos triángulos que dan forma de uno más grande), con las $46 \mathrm{~m}$ de radio máximo y $15 \mathrm{~m}$ de mínimo. Además, se presentan los límites teóricos para cada una de las tres curvas de dispersión generadas, con el fin de comparar el rango de frecuencias en que el arreglo genera información confiable. Cuando se habla de promedio de arreglos, se refiere a que la curva de dispersión es generada al promediar dos o más curvas de arreglos individuales obtenidos en diferentes tiempos de inicio de la medición, correspondientes a distintos radios. En este caso de análisis, los resultados en los tres sitios en estudio fueron bastante consistentes, por lo que se presenta únicamente los obtenidos en La Sabana.

Respecto a la variación en configuraciones geométricas, ejemplificada mediante los resultados obtenidos en La Sabana y presentados en la figura 4, se observa que el arreglo promedio de ocho sensores en radio grande y radio intermedio (46 m y $23 \mathrm{~m}$ respectivamente) muestra una amplitud mayor en su respuesta teórica. Los límites del rango seguro de dicho arreglo básicamente engloban los de los demás configuraciones estudiadas (promedio de radio grande e intermedio de seis sensores y triángulo de siete sensores). En cuanto a la configuración en triángulo, se obtiene información de frecuencias altas, contrario al arreglo promedio circular de seis sensores, que lo hace en bajas frecuencias. Esto se debe a que el arreglo en triángulo tiene un radio interno más pequeño que el del promedio de seis sensores (15 m para la configuración en triángulo y $23 \mathrm{~m}$ para el promedio de seis sensores). Lo anterior se explica en que los radios pequeños logran registrar mejor la dispersión de ondas de alta frecuencia correlacionadas con los estratos más superficiales, como se dijo antes.

En cuanto a la forma de las curvas de dispersión procesadas en la figura 4 , se observa que la mayor parte de estas son prácticamente iguales para las tres configuraciones geométricas. No obstante, en la zona de altas frecuencias, donde la curva de dispersión empieza a tender a una lentitud constante, las variaciones estudiadas tienden a separarse y muestran una alta desviación estándar, por lo que la verdadera trayectoria de la curva no se identifica con exactitud a partir de $\operatorname{los} 7 \mathrm{~Hz}$, aproximadamente.

\section{Cantidad de sensores utilizados en la medición}

En este apartado se va a estudiar la relación que tiene la curva de dispersión obtenida por la metodología F-K con la cantidad de sensores que se utilizan en la medición. En este caso, se consideran arreglos circulares con un radio aproximadamente del mismo tamaño, cuando el sitio lo permite, variando únicamente el número de sensores en el arreglo (ocho, seis y cuatro geófonos según el caso). Siempre se utiliza un sensor central y el resto en el perímetro. En la figura 5 se muestra la ubicación en el plano horizontal de los equipos según la cantidad de sensores utilizados.

En la figura 6 se presentan las curvas de dispersión obtenidas en el Parque Metropolitano La Sabana para las mediciones de ondas superficiales mediante arreglos circulares con apertura de $41 \mathrm{~m}$ de radio con ocho, seis y cuatro sensores. 


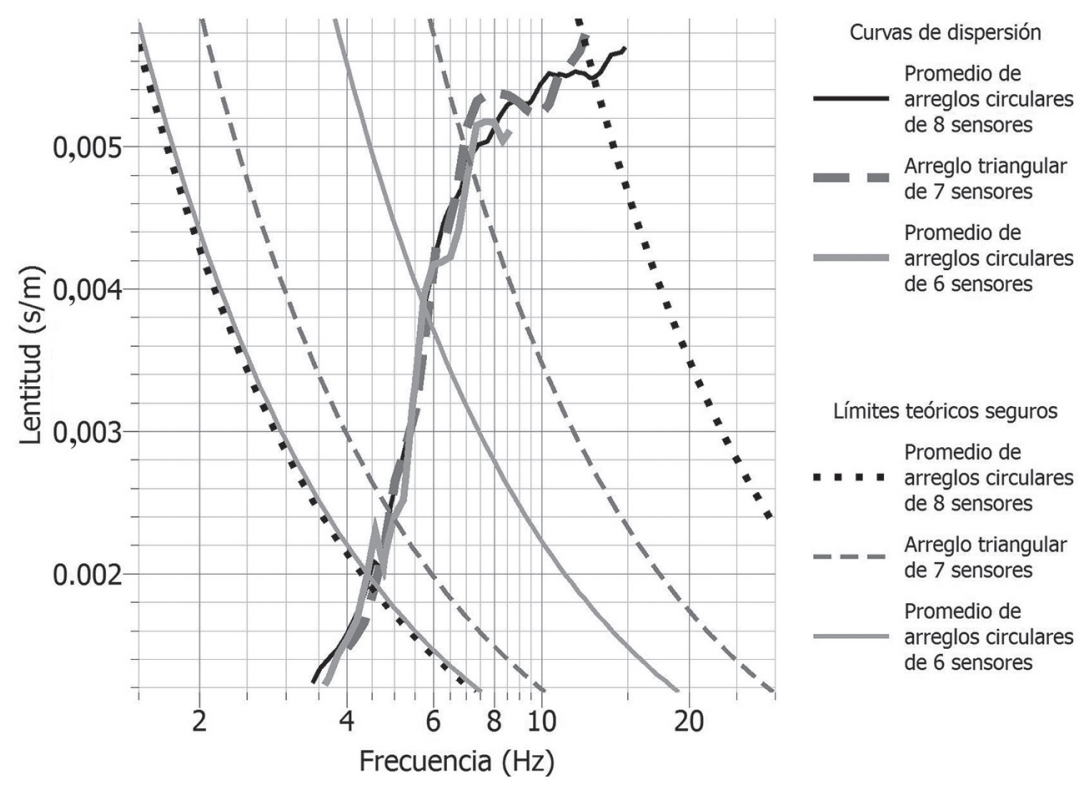

Fig. 4: Curvas de dispersión y rangos de respuesta teórica en el Parque Metropolitano La Sabana para el promedio de arreglos circulares de 8 sensores (radios de $46 \mathrm{~m}$ y $23 \mathrm{~m}$ ), arreglo triangular de 7 sensores (radios de $46 \mathrm{~m}$ y $23 \mathrm{~m}$ y arreglo circular de 6 sensores (radios de $40 \mathrm{~m}$ y $15 \mathrm{~m}$ ).

El efecto de la cantidad de sensores en las mediciones de ondas superficiales, mostrada en la figura 6, es una de las variaciones que más claramente se ve reflejada en las curvas de dispersión y en los límites de la respuesta teórica de los arreglos. El rango de frecuencias donde se tiene certeza de la información (rango seguro para la lectura de la curva) está estrechamente relacionado con la cantidad de sensores que se utilicen. Los resultados presentados en La Sabana, también son consistentes para los otros dos sitios considerados: en todos los casos la respuesta del arreglo de ocho sensores engloba a la de seis equipos y esta a su vez contiene a la de cuatro sensores, por lo que el número de geófonos guarda estrecha relación con la amplitud del rango de frecuencias en el cual se tiene información confiable del arreglo. Lo mismo sucede en los otros dos sitios estudiados, ya que la definición de los rangos dentro de los cuales la curva de dispersión es válida no depende del emplazamiento, sino del número y ubicación de sensores utilizados.

$\mathrm{Al}$ analizar las trazas en la Fig 6, mientras que en el arreglo de ocho sensores se logra apreciar la trayectoria de la curva de dispersión claramente entre los $3,5 \mathrm{~Hz}$ si y $10 \mathrm{~Hz}$ si se consideran los límites teóricos (Fig. 6a), en la medición de cuatro sensores no se logra apreciar adecuadamente la curva dispersión de puntos dentro de los límites teóricos (Fig. 6b). Es evidente que el rango definido por los límites seguros en el arreglo de cuatro sensores es sumamente pequeño, por lo que su uso se vuelve inapropiado (Fig. 6c).

\section{Apertura espacial en el arreglo de sensores}

Otra posible variación de la prueba de arreglos de sensores es el efecto de la apertura espacial en la curva de dispersión. Con base en lo expuesto anteriormente, el diámetro del arreglo, interpretado en esta investigación como la apertura, tiene una correlación directa con la profundidad de los estratos que se puedan estudiar. Los arreglos de mayor radio brindan mejor información de estratos más profundos mientras que los de menor apertura generan mejores modelos de las capas más someras. Por lo tanto, en muchas condiciones es recomendable combinar aperturas grandes y pequeñas para tener una información más 
completa del estrato de suelo que subyace. En la figura 7 se presentan las curvas de dispersión obtenidas en el Estadio Municipal de Palmares para las configuraciones circulares de ocho sensores con aperturas de $41 \mathrm{~m}, 20,5 \mathrm{~m}$ y 10,25 m de radio.

Según la teoría que respalda la metodología F-K, la apertura del arreglo incide directamente en los límites de la respuesta teórica y en los rangos de frecuencia donde se tiene la suficiente resolución en la curva de dispersión, lo cual se ve reflejado en los arreglos en el Estadio Municipal de Palmares presentados en la figura 7. En el caso de los radios más grandes, en todos los sitios se logra obtener la trayectoria de la curva en las bajas frecuencias de manera muy clara y con una desviación estándar bastante baja. Inclusive, en términos generales, el radio grande es el que brinda mayor información en un rango de frecuencias más amplio de la curva de dispersión, en comparación con las demás aperturas. Debido a las limitaciones de espacio en los lugares seleccionados para las pruebas, no se pudieron extender los arreglos más allá de los 100 metros de diámetro, por lo que información más profunda de los perfiles de suelo no pudo ser evaluada.

Al observar las curvas de dispersión del radio intermedio y el pequeño, lo más recomendable sería generar una única medición entre estas dos, utilizando un radio que sea el promedio de estos, ya que realizar tres mediciones de ondas superficiales en un mismo emplazamiento hace que las pruebas de campo resulten de mucha duración, por lo que sus costos aumentan.

En términos generales, se concluye que lo óptimo es realizar únicamente dos aperturas de arreglos, tomando en cuenta la relación beneficio/costo de las pruebas, ya que a partir de los tres sitios analizados, al utilizar arreglos por debajo de los $50 \mathrm{~m}$ de apertura se produce mucha información duplicada que no aporta mejoras ni a la curva de dispersión del sitio ni a los modelos de Vs en función de la profundidad que se obtienen. Se recomienda entonces realizar un arreglo con la apertura máxima que permita el equipo y el sitio seleccionado, para definir la forma de la curva en frecuencias bajas e intermedias y complementarlo con otro arreglo de la mitad o un tercio de la apertura máxima, para completar la curva de dispersión en altas frecuencias.

\section{CONCLUSIONES}

Con base en la aplicación de la técnica FK en los tres sitios seleccionados: Parque Metropolitano de La Sabana, Estadio de Palmares e Instalaciones Deportivas de la UCR, se observa que los resultados son bastante consistentes, en aspectos como los siguientes:

En cuanto al tiempo total del registro, a partir de mediciones de quince minutos se logra observar claramente la trayectoria de la curva de dispersión, por lo que se concluye que se debe cumplir con al menos este lapso de tiempo para poder aplicar correctamente la metodología F-K y obtener resultados confiable, para todas las aperturas y configuraciones consideradas.

Sin embargo, las barras que representan la desviación estándar para cada frecuencia aumentan para tiempos de medición de 5 y 15 minutos y disminuyen para mediciones de mayor duración, por lo que la ventana de tiempo que permite obtener un balance entre tiempo de la prueba y desviaciones estándar aceptables (barras de error pequeñas) es la correspondiente a 30 minutos.

Con base en resultados consistentes en los tres sitios, la mejor configuración de sensores estudiada es la circular con un sensor central y el resto perimetrales, haciendo uso de la mayor cantidad de equipos disponibles. Además, se logran muy buenos resultados al combinar la curva que proviene de un radio grande y de uno pequeño para un mismo sitio (por ejemplo de $50 \mathrm{~m}$ y $25 \mathrm{~m}$ respectivamente), ya que de esta forma se obtiene una curva confiable en un mayor rango de frecuencias. Las mediciones para estas dos aperturas pueden hacerse en tiempos de inicio distintos, ya que así se logra utilizar todos los sensores en cada configuración.

La medición hecha a partir de una configuración de triángulo es útil cuando se va a realizar una única disposición de sensores para medir las ondas superficiales en el sitio (no dos o tres mediciones como se suele hacer en el caso de arreglos circulares). La configuración triangular permite combinar radios grandes, necesarios para obtener información de capas más profundas, y radios pequeños para estudiar los estratos más someros. Para lograr lo anterior, se recomienda que las relaciones 

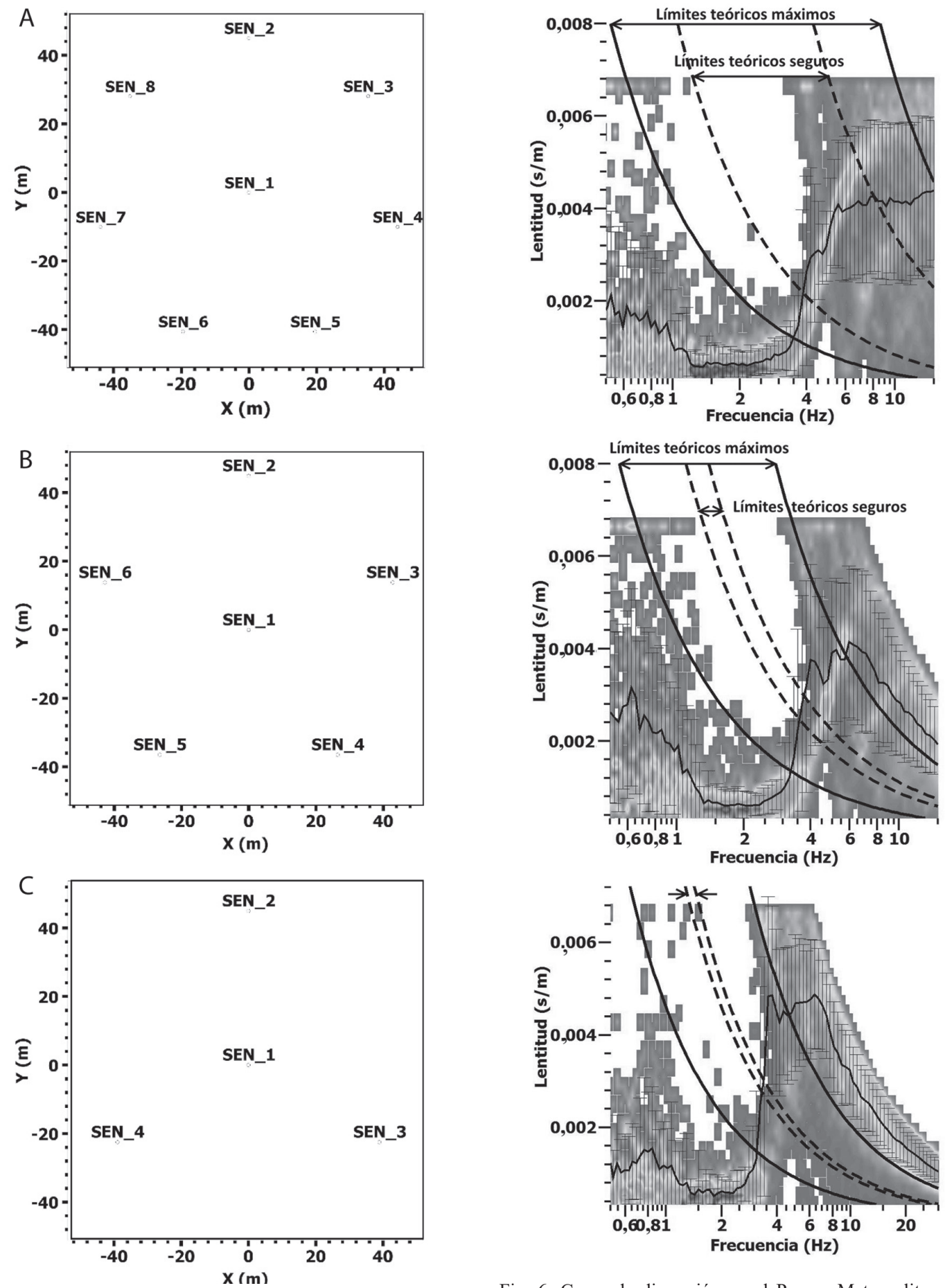

Fig. 5: Ubicación en planta de configuraciones circulares de (a) ocho sensores, (b) seis sensores y (c) cuatro sensores.

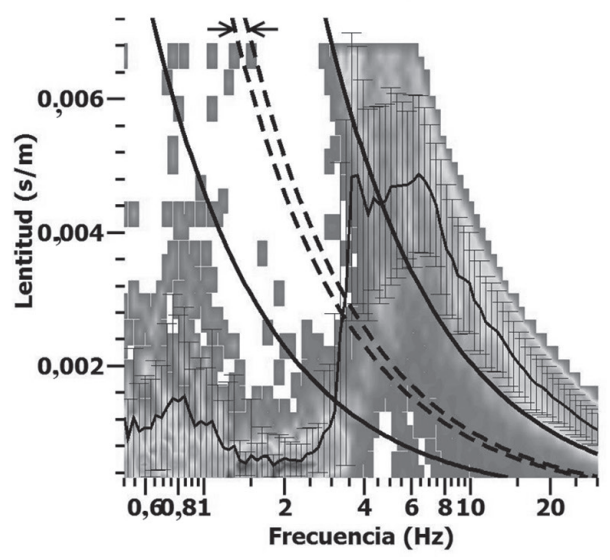

Fig. 6: Curva de dispersión en el Parque Metropolitano La Sabana, configuraciones circulares de $46 \mathrm{~m}$ de radio para (a) ocho sensores, (b) seis sensores y (c) cuatro sensores. 


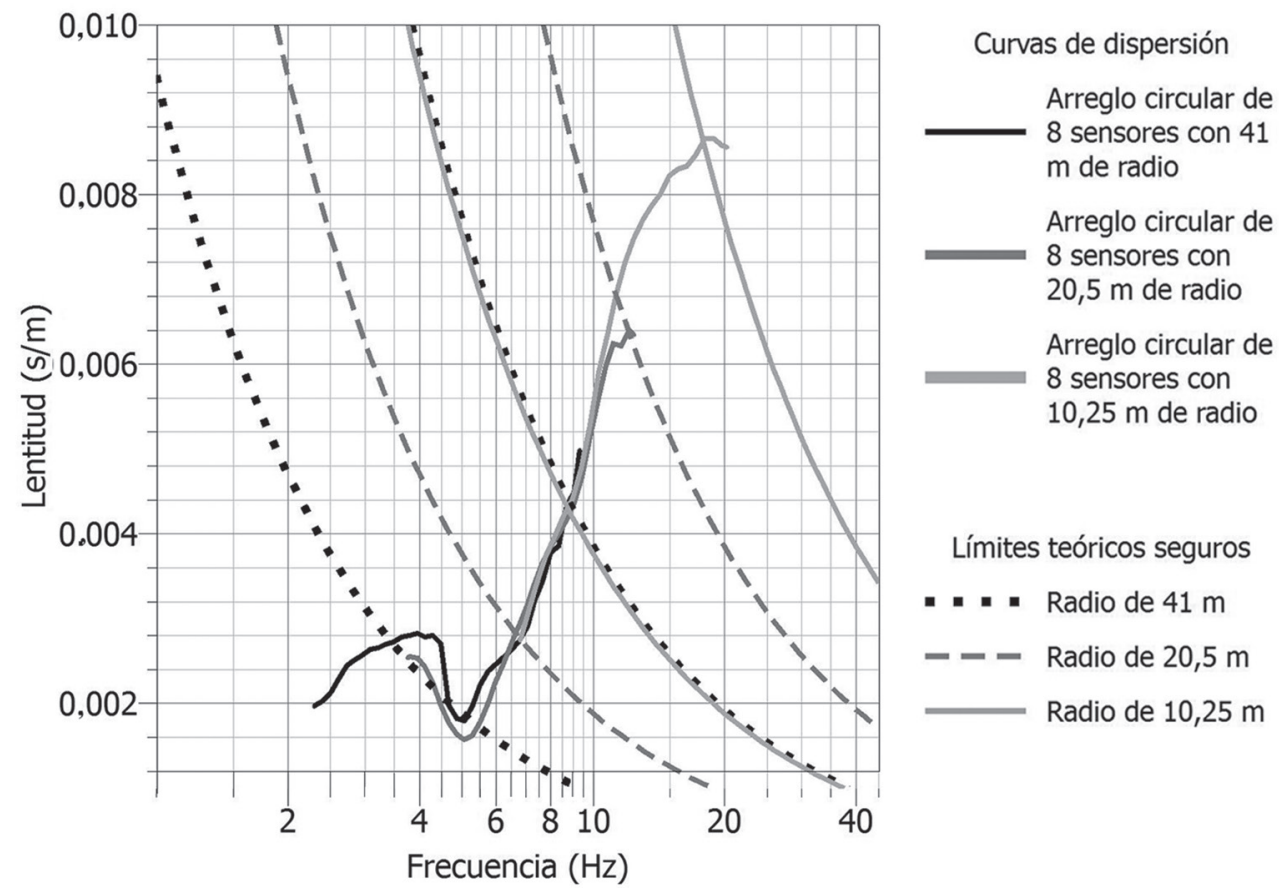

Fig. 7. Curvas de dispersión en el Estadio Municipal de Palmares para arreglos circulares de ocho sensores con radios de $41 \mathrm{~m}$, $20,5 \mathrm{~m}$ y $10,25 \mathrm{~m}$ junto con los límites teóricos.

entre el radio externo y el interno sean del doble o del triple. Por ejemplo, para un arreglo de seis sensores perimetrales y uno ubicado en el centro, si se utiliza una distancia de $60 \mathrm{~m}$ del central a los tres más alejados del triángulo, la distancia del central a los tres más cercanos debería ser de 30 mo menos.

En consistencia con lo observado por otros autores, la cantidad de sensores utilizados en las medición de ondas superficiales está directamente relacionado con la resolución y desviación estándar de la curva de dispersión en todo el rango de frecuencias, y la apertura en los límites teóricos del arreglo. En general, a mayor cantidad de sensores se obtienen mejores resultados de la prueba, debido a que se cubren más azimuts y se mejora la identificación de los arribos de los distintos frentes de onda.

En cuanto a la apertura de los arreglos de sensores, según las limitaciones de espacio para la colocación del equipo empleado, la configuración que brinda mayor información en el rango más amplio de frecuencias es el arreglo de mayor radio. Por lo tanto, se debe realizar siempre un arreglo con la máxima apertura que permita el sitio de la prueba y el equipo utilizado.

\section{REFERENCIAS BIBLIOGRÁFICAS}

SAUTER, F., 1989: Introducción a la Sismología.271 págs. Ed. Tecnológica de Costa Rica, Cartago.

SCHMIDT, V., 2010: Avances para estudios del riesgo sísmico a escala regional y local: aplicación a América Central y a la Bahía de Cádiz (sur de España).- 307 págs. Univ. Politécnica de Cataluña, Barcelona [Tésis Ph.D].

SCHMIDT, V., 2014: Clasificación de suelos de 15 estaciones acelerográficas mediante el uso de métodos basados en vibraciones 
ambientales y del parámetro Vs30.Rev. Geol. Am. Cent. (51): 33-67, DOI: 10.15517/rgac.v51i1.16850

SESAME EUROPEAN RESEARCH PROJECT, 2004: Guidelines for the implementation of the $\mathrm{H} / \mathrm{V}$ spectral ratio technique on ambient vibration. Measurements, processing and interpretations.- 62 págs. European Commission - Research General Directorate, Project No. EVG1CT-2000-00026 SESAME. [WP12 Report].
SESAME EUROPEAN RESEARCH

PROJECT, 2005: Site Effects Assessment Using Ambient Excitations.- 35 págs. European Commission - Research General Directorate, Project No. EVG1CT- 2000- 00026 SESAME. [WP13 Final Report].

WATHELET, M., 2007: Array recordings of ambient vibrations: surface-wave inversions.185 págs. Université de Liège, Bélgica. [Tesis Ph. D]. 